\title{
Sciatic Nerve Block in Single Nerve Block Technique for Unilateral Foot Surgery - an Alternative to Spinal Anaesthesia
}

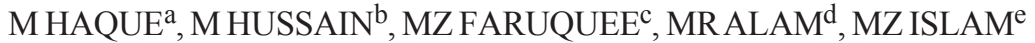

\begin{abstract}
Summary:
Background: In lower extremity surgeries, central neuraxial block or peripheral regional anesthesia technique can be used, mainly in elderly patients. This study investigates the efficiency of spinal anesthesia and sciatic nerve block techniques in lower extremity surgery. Spinal anesthesia may impair hemodynamic stability; peripheral nerve blocks targeting the sciatic nerve may be a useful alternative.
\end{abstract}

Objective: To compare Unilateral Spinal Anesthesia versus Popliteal Block in patients undergoing elective foot surgery to determine the method of better outcome.

Patients and Methods: This randomized comparative study was carried out on fifty co-operative patients of both sexes who were scheduled for elective foot surgeries. According to the used method of regional anesthesia, patients were divided into: (S) group unilateral intrathecal block with low-dose (7.5mg) of hyperbaric bupivacaine plus intrathecal fentanyl (25 mcg) and (P) group in which the sciatic nerve at the popliteal fossa was blocked via posterior approach by injecting $20 \mathrm{ml} 0.5 \%$ bupivacaine (100mg). The difficulty of the block performance, level of patient discomfort, block performance time, onset of sensory and motor blocks, time in hours to the first request for supplemental systemic analgesia postoperatively, its total consumption for 24 hours

Introduction:

Lower limb surgery under neuraxial block is a popular choice of technique. But many a time patients present

a. Prof. (Brigadier General) Mozibul Haque, Head of the department of Anaesthesiology, Combined Military Hospital, Dhaka

b. Colonel Muhammad Delwar Hussain, Classified specialist in Anaesthesia, Combined Military Hospital, Dhaka.

c. Dr. Md. Zulfiker Ali Faruquee, Consultant \& Associate Professor Cardiac Anaesthesia, Ibrahim Cardiac Hospital \& Research Institute, Shahbag, Dhaka

d. Lieutenant Colonel Md. Rabiul Alam, Classified specialist in Anaesthesia, Combined Military Hospital, Dhaka.

e. Major Md. Zahedul Islam Classified Anaesthesiologist, Combined Military Hospital, Dhaka.

Address of Correspondence: Professor (Brigadier General) Mozibul Haque, Head of the department of Anaesthesiology, Combined Military Hospital, Dhaka. Mobile: +8801769014756 , Email: colmhaque@yahoo.com

Received: 15 Sept., 2018

Accepted: 16 July, 2019 postoperatively and associated side effects were recorded in each group.

Results: Statistically, it was found no significant differences between the demographic characteristics as well as the duration of surgery between the groups. The groups did differ significantly in the difficulty of the block performance. However, a longer duration for performing the block was observed in the P group. The level of patient discomfort was significantly lesser in the $P$ group. The onset of complete sensory block was significantly longer in the P group. Hemodynamic profiles of our patients were found to be remarkably stable throughout the intraoperative period. In the P group, the time to first pain medication was significant longer. Moreover, the total dosage of analgesics during the first 24 hours postoperatively in group $P$ was highly significant lesser compared to the other groups.

Conclusion: Sciatic nerve block at the popliteal fossa is an ideal alternative where it is preferable to avoid spinal anesthesia for foot surgeries in haemodynamicaly unstable patients.

Key Words: Sciatic nerve block, spinal anesthesia, foot surgery.

(J Bangladesh Coll Phys Surg 2019; 37: 181-185)

DOI: http://dx.doi.org/10.3329/jbcps.v37i4.43347

with the conditions that preclude neuraxial anaesthesia relatively or absolutely. For example spinal anaesthesia in patients with severe aortic or mitral stenosis and cardiomyopathy with low ejection fraction is likely to cause profound hypotension. Neuraxial block is also relatively contraindicated in patients taking oral anticoagulant like warfarin and antiplatelet drug like clopidogrel. Other conditions that preclude neuraxial block are local sepsis, anatomical deformity of the spine and critically ill patient. So when patients present with this condition for lower limb surgery the next safer option is peripheral nerve block technique. Peripheral nerve block with a long acting local anaesthetic agent like bupivacaine not only provides surgical anaesthesia but also produces prolonged post-operative analgesia ${ }^{1}$. This avoids the adverse effects of opioids and NSAIDs when administered for postoperative analgesia. On the basis of the anatomical distribution of the sciatic nerve, 
block anywhere above its division into tibial and common peroneal nerve should produce complete surgical anaesthesia of the foot below the ankle. Although a significant mass of local anaesthetic is required to block sciatic nerve, there have been few reports of systemic drug disposition ${ }^{2}$. We therefore, designed a prospective study to assess the clinical effectiveness of sciatic nerve block alone for unilateral foot surgery below the ankle in comparison to spinal anaesthesia.

\section{Patients and methods:}

After getting ethical clearance fifty adult patients of ASA 1-3 presenting for different types of foot surgery below ankle were selected for the study. Informed consent was taken from each patient explaining the procedure of either spinal anaesthesia or sciatic nerve block in details. This study was conducted at the department of Anaesthesia, Combined Military Hospital, Dhaka over a period of 8-months. Patients who were willing to go for surgery whilst awake under sciatic nerve block were included for the study. No premedication was given to any patient before coming to operation theatre. On arrival in the anaesthetic room a $20 \mathrm{G}$ cannula was placed in the peripheral vein for the administration of fluid and drugs. On the operating table, fentanyl 50 microgram was administered intravenously before the placement of the block. Patients were divided into a spinal anesthesia (S) group and sciatic nerve block (P) group consisting of 25 patients in each group. Base line recording of heart rate, blood pressure and $\mathrm{SpO}_{2}$ were done non-invasively using standard monitoring technique, and then continued at $10 \mathrm{~min}$ intervals till the end of surgery. In the (S) group, the patients were positioned in lateral decubitus. The region was aseptically cleaned and draped. In the selected intervertebral space (L4-L5 or L3-L4) injection site, 2-3 $\mathrm{ml}$ of $1 \%$ lidocaine was injected into the skin and subcutaneous tissue. After ensuring that dura was passed and the spinal space was entered, the plunger of a $25 \mathrm{G}$ Quincke spinal needle was drawn back and the free flow of the spinal fluid was observed. $1.5 \mathrm{ml}$ of $0.5 \%$ hyperbaric bupivacaine was injected to the subarachnoid space. When this procedure was completed, the patient was kept in the lateral position for 5 minutes to ensure unilateral neuraxial block. In the (P) group, after infiltration of skin and subcutaneous tissue with $1 \%$ lignocaine, the sciatic nerve injection was performed at the popliteal fossa of the affected lower extremity. Confirmation of the needle placement was achieved by eliciting parasthesia and numbness along the course of the sciatic nerve. After careful intermittent aspiration, $20 \mathrm{ml}$ of $0.5 \%$ bupivacaine was injected over a period of $2 \mathrm{~min}$. All the blocks were performed by the same investigator.

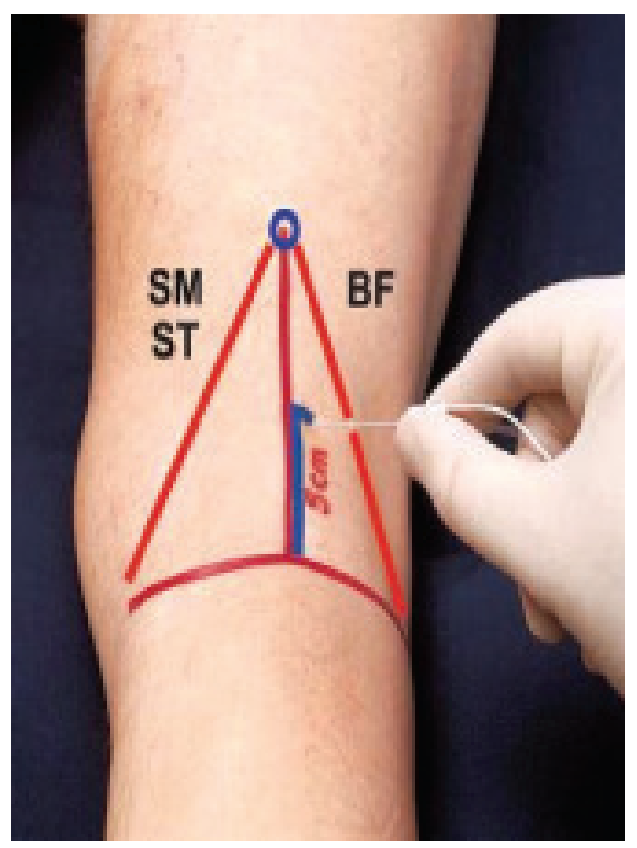

Fig.-1: Diagramatic presentation of Sciatic Nerve Block at the level of the popliteal fossa (SM Semimembranosus muscle, ST-Semitendenosus muscle, BF-Tendon of Biceps femoris)

Development of the sensory nerve block was assessed by the response to pin-prick on dorsal and planter aspects of the foot using a short-beveled $27 \mathrm{G}$ dental needle before and $5,10,15,25$, and 30 min after injection . Sensation was categorized as 'sharp' (same as the contra-lateral foot), 'dull' (pin-prick perceived as pressure) or 'absent' (complete loss of awareness of pinprick). Onset of sensory block was defined as the time taken to achieve complete loss of sensation ('dull'/ 'absent') on both dorsal and planter aspects of the foot. Once the sensory block was completed an arterial tourniquet was applied to the calf, a one hand's breadth below the tibial tuberosity to avoid proximal compression of the peroneal nerve. The foot was exanguinated using an Esmarch bandage, and the arterial tourniquet was inflated. The clinical efficiency of the 
sensory block was assessed by the use of Bromage Grading:

Excellent- complete surgical anaesthesia without use of i.v. supplementation

Good- surgery was possible with the use of i.v. opioids/ ketamine \& sedatives

Fair- inhalational anaesthetic was required in addition to i.v. supplementation.

The duration of sensory block was indicated as the onset of complete sensory block to the time that the patient first requesting for the post-operative analgesia. The patient's satisfaction was categorized on verbal rating as 'excellent' (very happy in all respect), 'good' (happy, no complain), 'fair' (not unhappy, it's OK) \& 'poor' (unhappy, could have been better). Each patient was observed for any untoward effect like nausea, vomiting, arrhythmia or seizure during the operation and in the postoperative period.

Statistical analysis was done using Statistical Software, SPSS (Statistical Package for Social Science) version 19. Data was presented as mean (standard deviation), median (interquartile range) and percentage values. Comparison of mean values was done using the t-test, with comparison of median values was performed using Kruskal-Wallis test. Percentage comparison of 'positive' and 'negative' values (response) was done using Pearson's Chi-squared test or by Fisher's Exact test as appropriate. Statistical comparison for the characteristics of the nerve block was performed using the MannWhitney $U$ test. Time to onset and duration of sensory block was compared by Wilcoxon signed rank test. The level of significance was set at $\pm=0.05(\mathrm{p}<0.05)$.

\section{Results:}

Statistically, there were no significant differences between the demographic characteristics (age, sex, weight and height) as well as ASA grading between the groups (Table 1). Statistically, the groups did not differ significantly in the difficulty of the block performance. But the mean block performance time in the $P$ group was highly significantly longer than that of the other group (Table 2). The majority of the patients in the groups needed only one or two attempts for either subarachnoid space identification or sciatic nerve localization while, the number of those who needed three attempts were 1 and 3 patients in group S and P respectively. Patient's discomfort, like shivering, nausea and vomiting were more common with spinal (S) group than sciatic (P). While technical difficulties like, vessel puncture and multiple attempts were more with sciatic nerve block (P) group than spinal (S) (Table III).

Statistically, there were no significant differences found between the demographic characteristics of the two groups like age, sex, weight, height and ASA grading (Table 1).

Table-I

The demographic characteristics of the patients

\begin{tabular}{lccc} 
& Spinal (S) group & Sciatic nerve block (P) group & P Value \\
\hline Age & $64.59 \pm 9.1$ & $62.59 \pm 8.7$ & 0.61 \\
Sex $(\mathrm{F} / \mathrm{M})$ & $7 / 18$ & $5 / 20$ & 0.74 \\
Weight $(\mathrm{kg})$ & $74.02 \pm 6.18$ & $73.67 \pm 7.66$ & 0.47 \\
Height $(\mathrm{cm})$ & $167.78 \pm 6.78$ & $171.31 \pm 5.32$ & 0.81 \\
ASA Grading (I/III) & $11 / 14$ & $8 / 17$ & 0.15 \\
\hline
\end{tabular}

Table-II

\begin{tabular}{lccc}
\multicolumn{5}{c}{ Onset of anaethesia, duration of surgery and duration of analgesia in both the groups } \\
& Spinal (S) group (min) & Sciatic nerve block (P) group (min) & P Value \\
\hline Onset of anaesthesia & $5.3 \pm 1.3$ & $8.9 \pm 1.8$ & $\hat{A} 0.001$ \\
Duration of surgery & $69.84 \pm 15.37$ & $70.13 \pm 13.67$ & 1 \\
Duration of analgesia & $185.20 \pm 37.65$ & $327.56 \pm 43.32$ & $\hat{A} 0.001$ \\
\hline
\end{tabular}

Data expressed as mean \pm SD

Differences are statistically significant when P value Â0.05

Both onset and duration of anaesthesia were significantly longer in sciatic nerve block group than spinal group. 
Table-III

\begin{tabular}{lcc}
\multicolumn{3}{c}{ Complications during the procedure } \\
& Spinal (S) group $(\mathrm{n}=25)$ & Sciatic nerve block (P) group (n=25) \\
\hline Vessel puncture & $1(4 \%)$ & $3(12 \%)$ \\
Shivering & $8(32 \%)$ & $1(4 \%)$ \\
Multiple attempt & $1(4 \%)$ & $3(12 \%)$ \\
Nausea, Vomiting & $2(8 \%)$ & $0(0 \%)$ \\
\hline
\end{tabular}

The majority of the patients in the groups needed only one or two attempts for either subarachnoid space identification or sciatic nerve localization while, the number of those who needed three attempts were 1 and 3 patients in group S and $\mathrm{P}$ respectively.

\section{Discussion:}

This study was designed to assess the clinical efficiency of sciatic nerve block alone as the primary anaesthesia for the foot surgery in comparison with traditional unilateral subarachnoid block. In our present study we found that the sciatic nerve block is a useful technique for unilateral foot surgery in terms of effective surgical anaesthesia and haemodynamic stability. In a prospective study on 100 patients, Davies et al showed that sciatic nerve block had an acceptable success rate of $89 \%$ and is a suitable technique for vascular surgical patients undergoing procedures distal to the knee joint ${ }^{3}$. The attractiveness of the sciatic nerve block lies in its potential ability to minimize haemodynamic disturbances and improve regional blood flow to the lower limb ${ }^{4,5}$. Fanelli et al compared the haemodynamic changes induced by combined sciatic-femoral nerve block and unilateral spinal anaesthesia. They found that the mean arterial pressure did not change in patients in nerve block group whereas in spinal group mean arterial pressure was reduced with a mean $15 \%$ reduction. Cardiac index was decreased by $15-20 \%$ in group spinal anaesthesia while no changes were observed in group nerve block ${ }^{6}$.

In this study we used $20 \mathrm{ml}$ of $0.5 \%$ bupivacaine and observed that mean duration of analgesia including effective surgical anaesthesia was 12 hours 30 minutes. In another study Tharwat used similar volume of $0.5 \%$ bupivacaine in combined femoral-obturator-sciatic nerve block for ACL reconstruction and found that the mean duration of analgesia was 14 hours and ranged up to 24 hours $^{9}$ which is comparable to our study. To produce effective sciatic nerve block for surgical anaesthesia we used $20 \mathrm{ml}$ of bupivacaine in a concentration of $5.0 \mathrm{mg} / \mathrm{ml}$. We did not notice any adverse systemic toxicity of bupivacaine such as seizure, arrhythmia, or cardiovascular collapse. Connolly et al used similar volume and concentration of bupivacaine to see the plasma concentration after sciatic nerve block and observed mean plasma and individual highest plasma concentration of 0.6 and $1.1 \mathrm{mg} /$ liter respectively ${ }^{8}$. Moore and colleagues observed a peak plasma concentration of $1.6 \mathrm{mg} /$ liter after $400 \mathrm{mg}$ bupivacaine with epinephrine for combined sciatic, femoral, and lateral cutaneous nerve of the thigh block ${ }^{9}$. The foregoing reviews attest the fact that even after use of significantly large mass of bupivacaine for peripheral nerve block its plasma concentration always remains well below its toxic level.

Adverse effects of the sciatic nerve block are rare, but our present study included some technical complications like multiple attempts and a few vessel punctures. We observed some minor complications like nausea, vomiting and shivering, due to the effect of spinal anaestesia.

\section{Conclusion:}

Sciatic nerve block alone provides good surgical anaesthesia and prolongs satisfactory postoperative analgesia. With the added advantages of stable haemodynamics and improved regional blood flow, sciatic nerve block should be considered more often in high risk patients undergoing foot surgery who are otherwise relatively contraindicate for spinal anaesthesia. Moreover sciatic block technique is suitable for day-stay surgery and meets the discharge criteria within several hours of surgery.

\section{References:}

1. Dr. Bindi B. Palkhiwala, Dr. Pauravi T. Bhatt. Study of combined femoral and sciatic nerve blocks for lower limb surgical procedures. GMJ 2015; 70(1): 36-40 
2. Misra U, Pridie A, McClymont C, Bower S. Plasma concentration of bupivacaine following combined sciatic and femoral 3-un-1 nerve blocks in open knee surgery. $\mathrm{Br}$ $J$ Anaesth 1991; 66: 310-3

3. Davies MJ and McGlade DP. One hundred sciatic nerve blocks: a comparison of localisation techniques. Anaesthesia intensive care 1993; 21:76-78

4. Laiq N, Khan MN, Khan S, Jan AS. Sciatic nerve block for lower limb surgery in elderly patients. JPMI 2005; 19(3): 256-60

5. Chia N, Low TC, Poon KH. Peripheral nerve blocks for lower limb surgery - a choice anaesthetic technique for patients with a recent myocardial infarction? Singapore Med $J$ 2002; 43(11): 583-586
6. Fanelli G, Casati A, Aldeghri G, Beccaria P, Berti M, Leoni A, Torri G. Cardiovascular effects of two different regional anaesthetic techniques for unilateral leg surgery. Acta Anaesthesiologica Scandinavia 1998; 42(1): 80-4

7. Tharwat A I. Combined posterior lumber plexus-sciatic nerve block versus combined femoral-obturator-sciatic nerve block for ACL reconstruction. Local and Regional Anesthesia 2011; 4 : 1-6

8. Connolly C, Coventry DM, Wildsmith JAW. Double-blind comparison of ropivacaine $7.5 \mathrm{mg} \mathrm{ml}^{-1}$ with bupivacaine 5 $\mathrm{mg} \mathrm{ml}^{-1}$ for sciatic nerve block. Br J Anaesth 2001; 86(5): 674-7

9. Moore D, Mather L, Bridenbaugh L, Balfour R, Lysons D, Horton W. Arterial and venous plasma level of bupivacaine following peripheral nerve block. Anesth Analg 1976; 55: $763-8$ 